\title{
Commonly used terminology in oral surgery and oral medicine: the patient's perspective
}

\author{
Alice Hamilton, ${ }^{* 1}$ Philip Lamey, ${ }^{2}$ Aman Ulhaq ${ }^{3}$ and Eleni Besi ${ }^{4}$
}

\section{Key points}

Gives an insight into terminology that has the potential to cause confusion or concern to patients.
Highlights how demographics and first language can influence health literacy.

\begin{abstract}
Introduction Dentistry is progressing into person-centred care and away from a paternal approach. Effective verbal and written communication are crucial to allow this collaboration; however, misunderstanding of terminology can lead to confusion, poor decision-making and poor health outcomes.
\end{abstract}

Methods A voluntary questionnaire with multiple-choice and short-answer questions was given to patients attending the NHS Lothian Oral Surgery and Oral Medicine Department over two weeks; 137 were completed. Eighteen terms were assessed, including: ulcer, local anaesthetic, impacted tooth, radiograph, sedation, biopsy, mucosa and benign. Exclusion criteria were non-English speakers who required a translator.

Results The multiple-choice questions revealed that terms such as 'blister' and 'local anaesthetic' are relatively well understood. Other terms, such as mucosa, were poorly understood. Over a third of patients confused 'sedation' with general anaesthetic. Short-answer questions revealed a wide range of answers. 'Biopsy' and 'radiograph' were generally better understood compared to other terms. Demographics, educational background and English as a first language appeared to have an influence on understanding.

Conclusion Patients had a varied understanding of terminology. Incorrect interpretation of words may lead to illinformed decision-making or unnecessary concern. It is essential that challenging terminology is identified and explained at an understandable level.

\section{Introduction}

Dentistry is progressing into person-centred care and away from the traditional paternalistic approach, as patients show an increased wish for more involvement in decision-making and treatment planning. ${ }^{1,2}$ Both effective verbal and written communication are crucial to allow this collaboration between healthcare professional and patient, as misunderstanding of information or terminology can cause unnecessary concern or confusion and lead to poor health outcomes. ${ }^{3}$ Oral surgery and oral medicine departments can be stressful and intimidating places, where patients may attend

${ }^{1}$ Clinical Research Fellow and Honorary Specialty Trainee in Restorative Dentistry, Dundee Dental Hospital, UK;

${ }^{2}$ Professor of Oral Medicine, Edinburgh Dental Institute, UK; ${ }^{3}$ Consultant in Orthodontics, Edinburgh Dental Institute, UK

${ }^{4}$ Consultant in Oral Surgery, Edinburgh Dental Institute, UK.

*Correspondence to: Alice Hamilton

Email address: alice.r.hamilton@gmail.com

Refereed Paper.

Accepted 7 September 2020

https://doi.org/10.1038/s41415-021-3073-1 regarding the surgical removal of a tooth or biopsy of a lump which they may fear is cancer. The misunderstanding of terminology in these settings could lead to worsening anxieties. ${ }^{3,4}$

The General Dental Council (GDC) standards expresses the importance of effective communication with patients, stating that dentists must give information in a way that the patient understands. ${ }^{5}$ Functional health literacy is the ability for the patient to understand and use health information, including terminology. ${ }^{6}$ High health literacy in patients can lead to increased levels of confidence in the professional and care provided. ${ }^{7}$ The importance and the need for good communication has also been stressed by the Academy of Medical Royal Colleges. ${ }^{8}$

Several papers have assessed the understanding of terminology in both dental and medical settings to gain a deeper understanding of patient comprehension, with the goal of improving patient-clinician communication. Previous areas assessed include oral medicine, ${ }^{9}$ cardiology, ${ }^{10}$ orthopaedics ${ }^{11}$ and breast clinic. ${ }^{12}$
The aim of this quality improvement project was to assess patients' understanding of commonly used terminology within the NHS Lothian Oral Surgery and Oral Medicine Department, allowing for improvements in both verbal and written communication between staff and patients, improving care and limiting unwarranted confusion or concern.

\section{Methods}

A voluntary piloted questionnaire consisting of multiple-choice questions (MCQs) and short-answer questions (SAQs) was offered to all patients attending the Oral Surgery and Oral Medicine Department in the Edinburgh Dental Institute over a two-week period, to assess patient understanding of commonly used terminology. A sample size of 100-150 patients, based on Hayes et al., ${ }^{9}$ was advised and approved by the Edinburgh Dental Institute Quality Improvement and Audit Team (EDIQIAT). This sample size would also fit the events per variable model for binary outcomes. ${ }^{13}$ Patients anonymously completed 
the questionnaire while waiting to attend their appointment; all ages were included. The questionnaire was adapted from the questionnaire used in Hayes et al. ${ }^{9}$ to include similar oral medicine questions, allowing for comparison while also expanding to include common oral surgery terminology considered by members of the Oral Surgery and Oral Medicine Department to be commonly used in verbal and written communication. The questionnaire was piloted on a small sample of patients, which was reviewed by the authors and approved by the EDIQIAT. Exclusion criteria were non-English speakers who required a translator.

The questionnaire was split into three sections. The first recorded patient demographics including age group, gender, education level, first language and whether a translator was required. Education level was assessed at secondary school, college, university or postgraduate levels. The second section had 11 MCQs assessing commonly used terms (Fig. 1; see online supplementary information). The final section asked patients to give their understanding of seven terms using SAQs.

\section{Results}

A total of 137 questionnaires were completed. Demographic results are shown in Table 1. There were 11 different first languages spoken between the 12 patients with English not as a first language.

\section{Multiple-choice questions}

Data analyses were performed using Prism version 8.4.3. Confidence intervals were set at $95 \%$ and $\mathrm{p}<0.05 \%$ was considered statistically significant.

In total, 137 patients answered all the MCQs. Figure 1 shows the percentage of correct, incorrect and 'don't know' answers for MCQs. For all patients, there was a mean correct MCQ score of 7.25 and a median of 8 . Only eight patients answered all 11 MCQs correctly, of which five were educated to postgraduate level, one to university level and two to college level. English was not the first language of one of these patients, who selected Russian.

All MCQ data was analysed using a ShapiroWilk normality test. This showed a nonGaussian distribution of data; therefore, the two-tailed Mann-Whitney U test was used to compare two datasets and the Kruskal-Wallis test to compare larger datasets (Table 1).
Fig. 1 Charts showing percentage of multiple-choice answers $(n=137)$
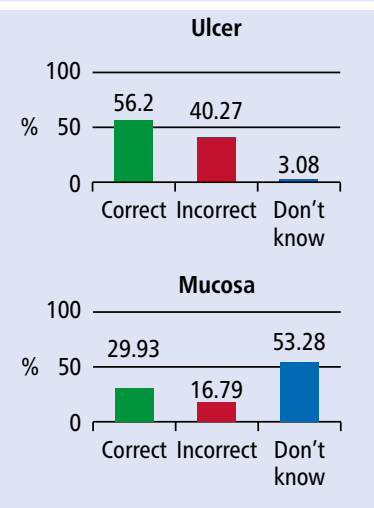

Local anaesthetic
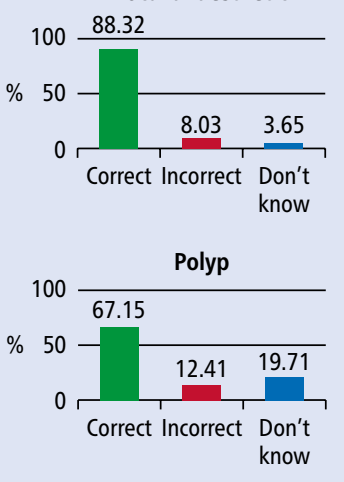
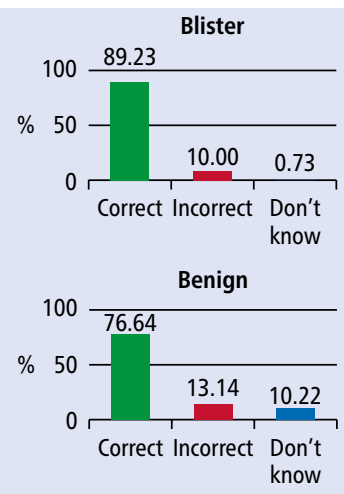

Sedation
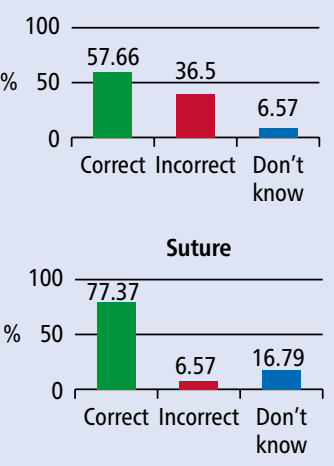
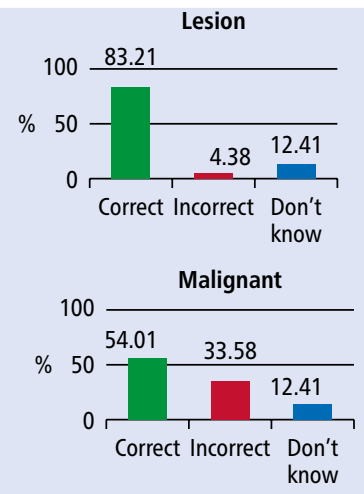

Cyst

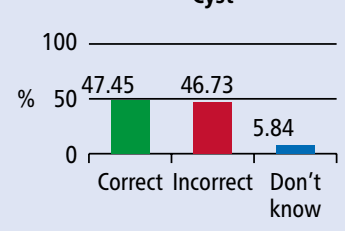

Table 1 Demographics showing comparison of gender, age group, education level and first language groups $(n=137)$

\begin{tabular}{|c|c|c|c|c|c|}
\hline \multicolumn{2}{|l|}{ Demographic } & \multirow{2}{*}{$\begin{array}{l}\text { Percentage } \\
58.4 \%(n=80)\end{array}$} & \multirow{2}{*}{$\begin{array}{l}\text { Median } \\
8\end{array}$} & $P$ value & \multirow{2}{*}{$\begin{array}{l}\text { Mean } \pm \text { SD } \\
7.4 \pm 2.68\end{array}$} \\
\hline \multirow{3}{*}{ Gender } & Female & & & \multirow{3}{*}{$\begin{array}{l}0.562^{*} \\
\text { Not significant }\end{array}$} & \\
\hline & Male & $31.4 \%(n=43)$ & 7 & & $7.2 \pm 2.10$ \\
\hline & Not specified & $10.2 \%(n=14)$ & 7.5 & & $6.9 \pm 2.77$ \\
\hline \multirow{6}{*}{ Age group } & Under 25 & $6.6 \%(n=9)$ & 7 & \multirow{6}{*}{$\begin{array}{l}0.246^{\star} \\
\text { Not significant }\end{array}$} & $5.8 \pm 3.38$ \\
\hline & $25-34$ & $21.9 \%(n=30)$ & 7 & & $6.3 \pm 2.23$ \\
\hline & $35-44$ & $13.1 \%(n=18)$ & 7 & & $7.1 \pm 3.09$ \\
\hline & $45-54$ & $16.1 \%(n=22)$ & 7.5 & & $7.6 \pm 1.53$ \\
\hline & $55-64$ & $19.7 \%(n=27)$ & 8 & & $7.9 \pm 2.35$ \\
\hline & $65+$ & $22.6 \%(n=31)$ & 8 & & $7.4 \pm 2.71$ \\
\hline \multirow{5}{*}{ Education level } & Secondary school & $26.3 \%(n=36)$ & 7.5 & \multirow{5}{*}{$\begin{array}{l}0.1509^{*} \\
\text { Not significant }\end{array}$} & $6.8 \pm 2.74$ \\
\hline & College & $19.0 \%(n=26)$ & 7 & & $6.7 \pm 2.65$ \\
\hline & University & $27.0 \%(n=37)$ & 8 & & $8.0 \pm 1.83$ \\
\hline & Postgraduate & $21.9 \%(n=30)$ & 8 & & $7.6 \pm 2.79$ \\
\hline & Not specified & $5.8 \%(n=8)$ & 7 & & $6.8 \pm 2.12$ \\
\hline \multirow{2}{*}{ Language } & $\begin{array}{l}\text { English as a first } \\
\text { language }\end{array}$ & $\begin{array}{l}91.2 \%(n= \\
125)\end{array}$ & 8 & \multirow{2}{*}{$\begin{array}{l}0.0039 * * \\
\text { Significant }\end{array}$} & $7.5 \pm 2.30$ \\
\hline & $\begin{array}{l}\text { English not as a first } \\
\text { language }\end{array}$ & $8.8 \%(n=12)$ & 5 & & $4.9 \pm 3.40$ \\
\hline Total patients & & $n=137$ & 8 & & 7.25 \\
\hline
\end{tabular}


Logistic regression

Multiple logistic regression was used to analyse how each of the following four variables affected higher-scoring patients if the other variables were kept constant: age, gender, English as a first language and education level (Table 2). Education level was split into a combined higher education group (university and postgraduate level) and a combined lower education group (secondary school and college level). Higherscoring patients were considered as those who correctly answered 8-11 MCQs, which was higher than the median score. Of the 137 patients, 21 were excluded due to missing data such as gender or education level.

Table 2 Multiple logistic regression for patients scoring 8-11 multiple-choice questions correctly $(n=116)$

\begin{tabular}{|c|c|c|c|c|c|}
\hline \multicolumn{2}{|l|}{ Variables } & Odds ratio & $\begin{array}{l}\text { Confidence } \\
\text { intervals }\end{array}$ & $P$ value & Significance \\
\hline Age & Same age groups as Table 1 & 1.291 & 1.008 to 1.670 & 0.0002 & Significant \\
\hline \multirow{2}{*}{ Gender } & Female & 3.134 & 1.280 to 8.210 & \multirow{2}{*}{0.0151} & \multirow{2}{*}{ Significant } \\
\hline & Male & Ref & Ref & & \\
\hline \multirow{2}{*}{$\begin{array}{l}\text { Education } \\
\text { level }\end{array}$} & $\begin{array}{l}\text { Lower education } \\
\text { (Secondary school/college) }\end{array}$ & Ref & Ref & \multirow{2}{*}{0.0050} & \multirow{2}{*}{ Significant } \\
\hline & $\begin{array}{l}\text { Higher education (University/ } \\
\text { postgraduate) }\end{array}$ & 3.687 & 1.530 to 9.620 & & \\
\hline \multirow[b]{2}{*}{ Language } & English as a first language & 22.21 & 3.629 to 434.9 & \multirow[b]{2}{*}{0.0053} & \multirow[b]{2}{*}{ Significant } \\
\hline & $\begin{array}{l}\text { English not as a first } \\
\text { language }\end{array}$ & Ref & Ref & & \\
\hline
\end{tabular}

Fig. 2 The ROC curve derived from the multiple logistic regression

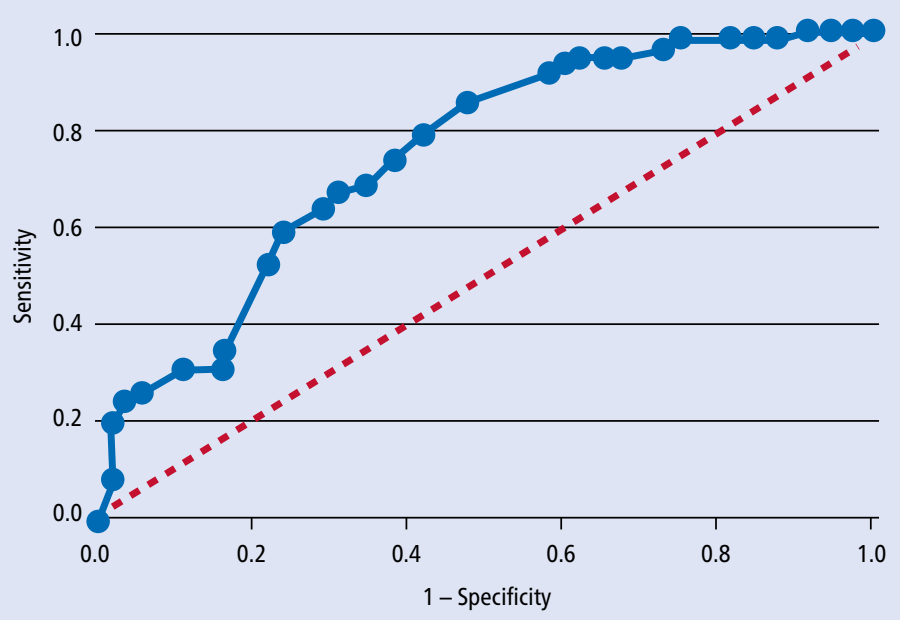

Fig. 3 Pie chart showing percentage of answers for the term 'biopsy' $(n=137)$

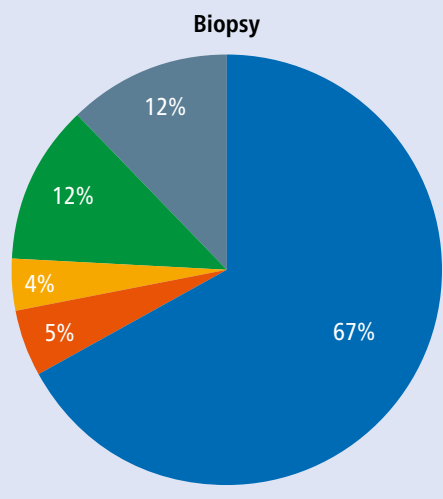

Removal of tissue sample

Removal of tissue sample to see if cancer or benign

Medical test

Other

Don't know

The receiver operating characteristic (ROC) curve cut-off value was 0.5 and the area under the graph was 0.749 ( $\mathrm{p}<0.001,95 \%$ CI 0.660 to 0.838) (Fig. 2).

\section{Short-answer questions}

Figure 3 shows the percentage of SAQ answers for 'biopsy'. Other definitions given for this term included scan, surgical procedure, body check-up and medical test.

Figure 4 displays the results for 'tumour'. Two patients also associated the word with a lump in the brain.

Figure 5 shows SAQ results for 'metastasis'. Other definitions given included infection caused by breast milk, something wrong in foot, soft lump, death of cells and a type of tumour.

Figure 6 shows percentages of some of the varied SAQ answers for 'impacted tooth'. Many other answers confused it with blunt force trauma, toothache, infection of gum or tooth, infected socket, procedure to a tooth, overcrowding and pushing a tooth back into the gum.

Figure 7 shows the definitions given for 'radiograph'. Two patients, however, confused 'radiograph' with the treatment of cancer. Other definitions were photo or picture, investigation in area that might be cancerous, CT/MRI, measure of damage to bone and treatment of cells that spread infection.

Definitions given for 'lymph node' are shown in Figure 8. Other definitions included skin tag, lump, cyst and growth on tonsils.

'Premalignant' SAQ results are shown in Figure 9. Other definitions included investigation of possible cancer, not a cancer, pre-diagnosis and stage before infection spreads to another part of the body.

\section{Discussion}

What is jargon and should we use it?

The dictionary defines jargon as 'words and phrases used by particular groups of people, especially in their work, that are not generally understood. ${ }^{14}$ Although GDC standard 2.3.3 states that dentists should avoid the use of jargon and acronyms, ${ }^{5}$ the literature shows that some patients feel more comfortable and confident with the use of medical and dental terminology in a move towards patient-centred care. ${ }^{3}$ The involvement in decision-making and increasing use of internet resources may lead to patients having a better understanding of terminology and 


\section{Fig. 4 Pie chart showing percentage of answers for the term 'tumour' $(n=137)$}

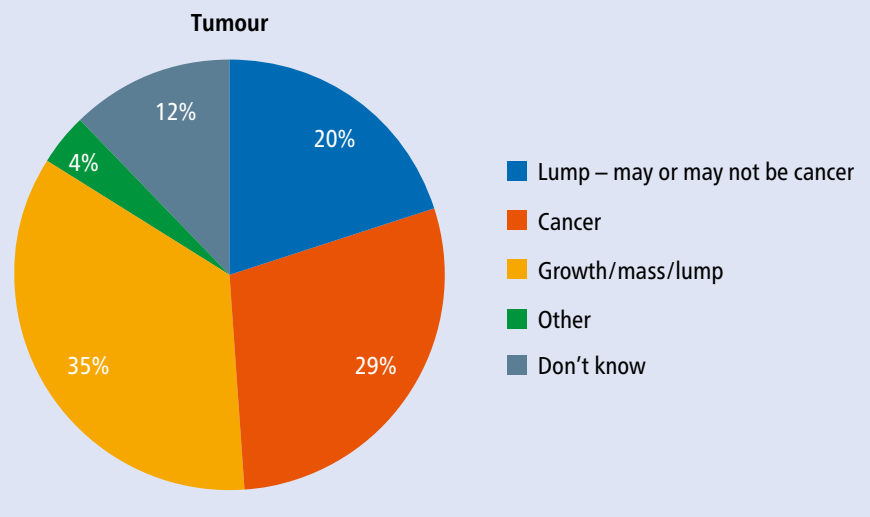

Fig. 5 Pie chart showing percentage of answers for the term 'metastasis' $(n=137)$

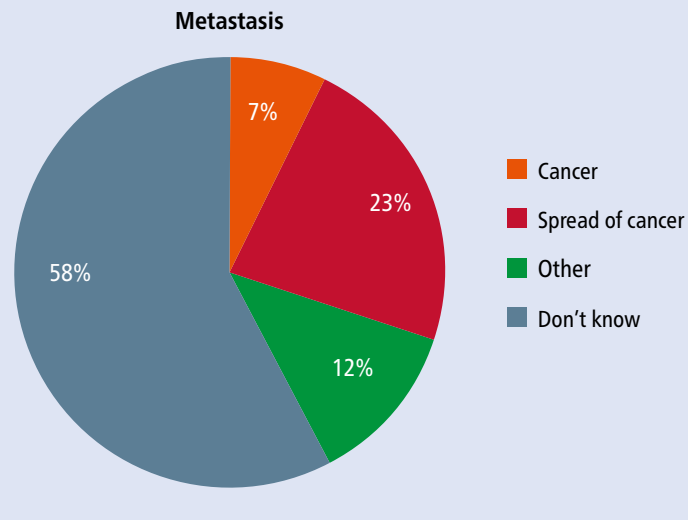

Fig. 6 Pie chart showing percentage of answers for the term 'impacted tooth' $(n=137)$

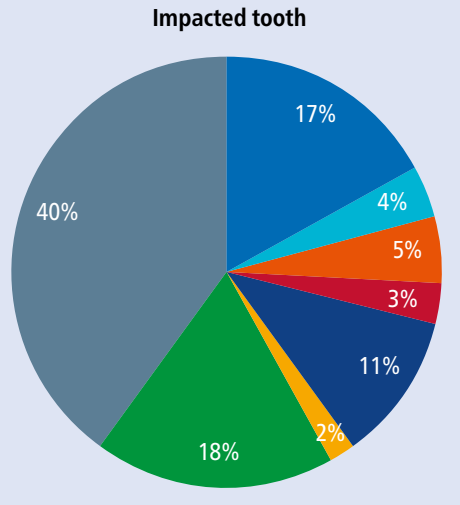

Does not come through gum/stuck under gum

Decay

Broken tooth/damaged tooth

Tooth too close to other teeth

Tooth growing in wrong position or angle

No room to come through

Other

Don't know

therefore a higher functional health literacy in the general population than in the past. $^{3}$ This can empower patients, leading to better engagement and participation in their care, ultimately resulting in better health outcomes. ${ }^{15,16}$

Some clinicians may argue that terms such as 'blister' and 'ulcer' are widely used and may not consider them jargon. The results for 'blister' may offer some support to this evaluated, as what might be assumed to be a commonly understood word may not turn out to be as such. For example, only $56 \%$ selected the correct definition of 'ulcer' (Fig. 1). This highlights that assumption of understanding should not be made on the basis of common use and stresses the need for clinicians to regularly establish patient comprehension of given information.

\section{Literacy in Scotland}

The Scottish Survey of Adult Literacy 2009 (SSAL) established that there are many potential factors associated with low literacy such as age, gender, socioeconomic factors, education and health. ${ }^{17}$ It identified that $26.7 \%$ of the population faced occasional literacy challenges, with 1 in 28 people facing serious challenges. There is a strong correlation between low literacy and low health literacy. However, it is important to remember that health literacy can also be low even in people with high general literacy skills. ${ }^{18}$

The results of this paper support that education level can be an influence for lower literacy, including health literacy, with significantly higher average correct MCQs in the combined university and postgraduate group compared to the combined secondary school and college group. Many patients are often good at hiding low literacy due to perceived shame. ${ }^{19}$ It is important for healthcare professionals to be aware of methods patients may employ to disguise low literacy, such as poor compliance, unfilled or partially filled forms, repetitively failing to attend appointments, excuses such as 'forgotten glasses' and confusion over medication name or instructions. ${ }^{20}$ To complete the questionnaire, there was already an assumed level of literacy; this could introduce some bias towards more literate patients.

The SSAL also showed that women aged below 55 years had stronger literacy skills than men in the same age group, but above 55 years, this picture reversed. The opposite was shown in our results, with females over 55 correctly identifying significantly higher average correct MCQs than all other groups with a median of $9(p=0.006)$. However, the literacy levels between genders compared to age are likely to have changed since the SSAL in 2009 , meaning many of those under 55 will now have moved into the higher age bracket, and this paper has a much smaller sample size than the SSAL. 


\section{Fig. 7 Pie chart showing percentage of answers for the term 'radiograph' $(n=137)$}

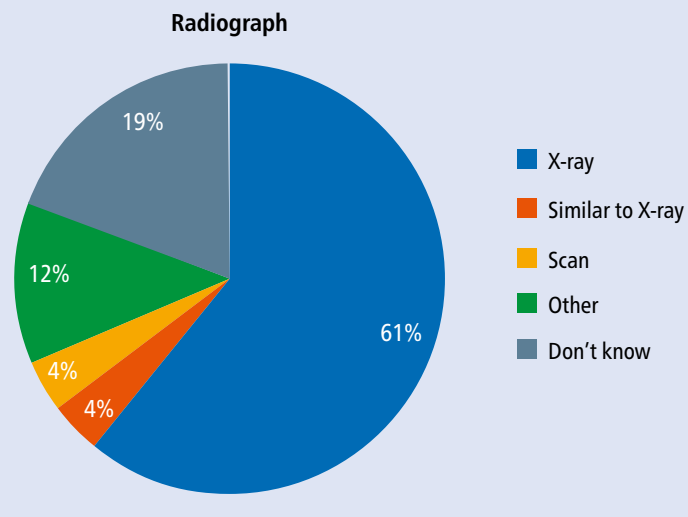

Fig. 8 Pie chart showing percentage of answers for the term 'lymph node' $(n=137)$

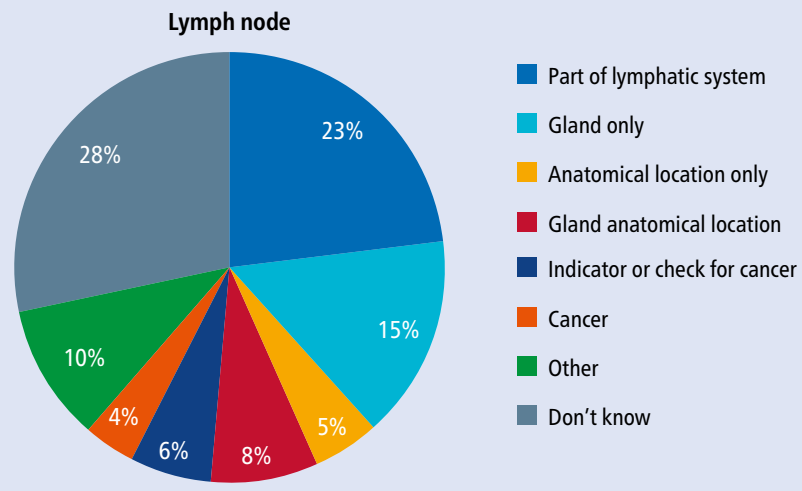

Fig. 9 Pie chart showing percentage of answers for the term 'premalignant' $(n=137)$

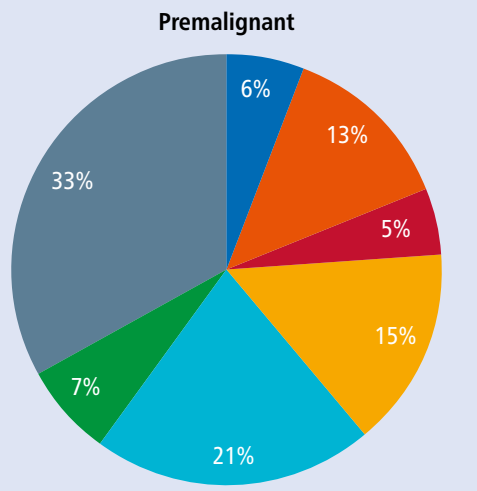

Before cancer

Cancer before it is a problem

Early cancer/cancer being reformed

Precancer

Not cancer - could become cancer

Other

Don't know

\section{Logistic regression}

Mann-Whitney U tests and Kruskal-Wallis tests on the independent variables of age, gender, education level and first language were carried out on all 137 patients (Table 1). Twenty-one patients had not completed gender or education level; therefore, multiple logistic regression was carried out on the 116 patients who had full datasets (Table 2). Unfortunately, this meant that some of the overall data was lost variables. This showed that patients with English as a first language remained the biggest predictor of a high score of 8-11 MCQs, with the odds being over 22 times more likely than for patients who did not. Many patients fluent in English (without it being their first language) may have good conversational English, but lack an understanding of medical or dental terms that they are less likely to have frequently come across. These patients may benefit from further explanation and gentle encouragement to accept a translator, even when they do not consider themselves to require one, in order to avoid communication errors. It is important to remember that patients without English as a first language accounted for a very small sample size and this may contribute to the large confidence intervals in the logistic regression (Table 2).

The multiple logistic regression also showed that females (OR 3.13), increasing age group (OR 1.29) and combined higher education group (OR 3.69) were also significant for predicting higher scores when all other variables were adjusted (Table 2).

\section{Verbal communication}

It has been suggested that between $40-80 \%$ of information provided to patients in an appointment is immediately forgotten. ${ }^{22,23} \mathrm{Ley}^{24}$ suggests that adherence to medical information relies on three factors: understanding, satisfaction and recall, where recall is directly affected by patient understanding. ${ }^{22}$ Where possible, handing over control of the appointment structure to the patient through patient-led consultations may increase recall of information and satisfaction, and lead to better compliance. $^{23}$

Effective verbal communication is essential for all aspects of patient care. A commonly misquoted theory regarding verbal communication is the 'Mehrabian model', oversimplified to imply that communication consists of $55 \%$ body language, 38\% tone and $7 \%$ meaning of spoken word. ${ }^{25}$ This generalisation suggests that words do not carry a heavy weighting; however, the studies involved looked at these aspects when conveying attitude and emotions, not information. ${ }^{26}$ Although important for emotive communication, the model does not apply to all verbal communication and must be used in context. When delivering important information such as diagnosis, treatment planning and advice, the understanding of words may carry higher value, especially 
when establishing valid consent. The model also cannot be directly applied to telephone conversation as this has no reliance on body language, nor to written communication where the weighting is almost entirely reliant on words. ${ }^{24}$

COVID-19 has brought new logistical and time-sensitive challenges to dental treatment. Increased personal protective equipment may mask facial expressions, losing important non-verbal cues normally required for the delivery of compassionate care. ${ }^{27,28}$ Effective verbal communication is therefore increasingly important, with an emphasis on words and tone to help reduce anxieties and maintain trust and patient safety.

Terms in the questionnaire which are often used in treatment planning discussions in oral surgery included 'local anaesthesia' and 'sedation'. 'Local anaesthesia' had a relatively high understanding, with $88 \%$ of patients selecting correctly (Fig. 1). This commonly used term in dentistry is often disguised from paediatric patients and becomes more directly used in communication with adults. Although a well-answered question, over one in ten patients didn't answer correctly. Even with the correct answer written in front of them, they confused 'local anaesthetic' with general anaesthetic, sedation or selected 'don't know'. There was an even higher misunderstanding of the term 'sedation', with only $58 \%$ of patients answering correctly and $34 \%$ confusing it with general anaesthetic. The misunderstanding of terms such as 'sedation' and 'local anaesthetic' could lead to serious consequences for the patient, potentially resulting in poor decisionmaking and invalid consent.

\section{Written communication}

'Mucosa' is a term that is less likely to be used in verbal clinician-patient communication and more likely in written communication, such as biopsy result letters. This was the least correctly answered MCQ, with less than a third answering correctly (Fig. 1). In hospital dentistry, it is common practice to write letters directly to the patient's general dental or medical practitioner. However, in a move towards person-centred care, writing letters directly to the patient has become a controversial topic. The Academy of Medical Royal Colleges published guidance in 2018 discussing the importance of writing letters to patients in order to keep them informed of the decision-making in their own health. ${ }^{8}$ High levels of patient satisfaction have been documented with this approach. ${ }^{29}$ However, there remains concerns among clinicians due to clinical time constraints and fears of potential misunderstanding of letter content. The results of this paper suggest that use of terms such as 'mucosa', 'sedation' or 'ulcer' without context or explanation may lead to unnecessary stress and confusion. It is therefore important that dental professionals appreciate the wants and needs of the patient, including their comprehension of terminology if writing letters to patients is to become part of common practice. Letters should be tailored to each patient's literacy level, ${ }^{4}$ with tools available via the NHS digital service manual to help increase the readability of letters. ${ }^{30}$

\section{Patient language}

Patients often have their own lexicon to describe dental terms. ${ }^{31}$ This paper allowed exploration of this through SAQs where patients could answer in their own words. An example of this different patient language was shown with 'radiograph' where patients answered using words such as 'x-ray', 'scan', 'picture' or 'photo'. This may be due to encountering descriptions beforehand from professionals, the media or patient-to-patient interaction. ${ }^{3}$

There were some interestingly similar malapropisms in both this paper and Hayes et al., ${ }^{9}$ with patients defining 'metastasis' as associated with either breastmilk or the foot, likely mistaking the term for sounding similar to 'mastitis' and 'metatarsal', respectively. A couple of patients also confused 'radiograph' with radiotherapy. It is important to remember that all the terms in the questionnaires were displayed as words without context; there is a possibility that there may be less misunderstanding when the term is used in a sentence.

'Impacted tooth' raised the largest variation of answers compared to other questions. Many patients correctly identified certain aspects associated with impaction such as a tooth growing in the wrong direction or stuck under the gum (Fig. 6). It is unknown how many patients previously attended an appointment in the department, as this was not assessed. The correct but varied answers may be due to descriptions heard when attending the department previously, from their own dentist or other sources of literature access between appointments to gain further insight. It has been suggested that health literacy varies, with understanding often stronger in areas which reflect the patient's own health concerns, ${ }^{3}$ and many of the $40 \%$ answering 'don't know' or not answering (Fig. 6) may have done so because this was not a term relevant to their own health. Many others wrote answers such as 'decay', 'infection, 'broken tooth' or described some form of treatment such as packing or extraction. Although these are not definitions of an impacted tooth, they are possibly words that patients have linked from past experience such as 'decay' of a third molar or adjacent tooth due to impaction, a 'broken tooth' due to caries in the third molar, or 'infection' and 'treatment' related to pericoronitis.

\section{Differences in literature}

There were more correct answers for 'benign' than 'malignant', with $77 \%$ and $54 \%$, respectively (Fig. 1). This contradicted the results of Hayes et al., where 'benign' was the least well-understood word (64\% correct answers) and 'malignant' was better understood (70\% correct answers). Another term with largely differing results when compared to Hayes et al. was 'lesion'. Our results showed $83 \%$ correct answers (Fig. 1), compared to only $65 \%$ shown by Hayes et al. The reason for these differences remains unknown; however, functional health literacy is always evolving, with increasing numbers of patients turning to sources such as the internet to gather information. ${ }^{3}$ Health literacy may also differ from region to region in the UK depending on different exposures to media and public health messages. ${ }^{32}$

\section{Associations with cancer}

'Biopsy' and 'tumour' were two of the most answered SAQs, both with only $12 \%$ of patients writing 'don't know' or not answering (Figures 3 and 4). Hayes et al. found that 29.1\% defined 'biopsy' as a test for cancer; however, our results found only $5 \%$ of patients supplied this answer. In fact, the results of this paper appeared to show a generally less cancer-phobic cohort than Hayes et al., with more patients identifying MCQ terms such as 'benign' or 'malignant' as 'not cancer' and appearing not to mention cancer as frequently in their SAQ definitions. ${ }^{9}$ An exception to this was perhaps 'tumour', with $29 \%$ of patients identifying it as cancer and a further $20 \%$ mentioning cancer but correctly identifying it as 'may or may not be cancer' (Fig. 4). This could not be compared to Hayes et al. as they did not separate the two in their results. 'Tumour' may not be a commonly used term for communication with patients; however, it is still important to retain 
the knowledge that, when used, it may create the connotation of cancer, as it did for almost a third of the patients.

Another word raising connotations of cancer was 'lymph node', with $4 \%$ identifying it as cancer and $6 \%$ describing it as a place to check for cancer (Fig. 8). Clinicians must be very careful to discuss that swollen lymph nodes can often present due to non-sinister causes. Thirty-six percent of patients correctly identified 'lymph node' as either part of the lymphatic system or a gland, sometimes including an anatomical location; however, some patients described anatomical locations alone, such as neck, armpit or breast. This may indicate that patients are aware of where lymph nodes might be situated but not of their function.

'Premalignant' was fairly well answered, with $42 \%$ writing 'precancer' or writing that it may become cancer. A further $18 \%$ described it as early or non-problematic cancer (Fig. 9). No patients associated it with a developed cancer, indicating that the term may not cause undue concern. However, a third did not answer the question or wrote 'don't know'; therefore, it cannot be ruled out that use of this word could cause confusion or concern without adequate definition or context.

\section{Limitations}

In hindsight, the multiple-choice answers for 'cyst' may have been too similar and more suited for dental school examination rather than for the purposes of this paper. Forty-seven percent identified it as 'abnormal area, usually fluidfilled' and a further $42 \%$ as 'lump of inflamed tissue' (Fig. 1). Although the latter is not the most correct answer, this misunderstanding is unlikely to cause issue. Similarly, $9 \%$ of patients confused 'polyp' with the definition for cyst; this incorrect understanding is unlikely to cause increased concern or errors in treatment (Fig. 1). However, the misunderstanding of 'polyp' as infection in $4 \%$ of patients could lead to different treatment decisions.

The results provided an insight into the current general understanding of terminology of patients in our waiting room. This has aided clinicians to help adapt and pitch their language at an appropriate level to support a person-centred approach. An adaptive style is required, where patients' abilities and understanding are established, allowing for information to be appropriately tailored. ${ }^{3,9,18}$ This has its challenges, especially within the oral surgery department, where patients are often managed by different members of the team throughout their patient journey. This lack of continuity may result in less time for the establishment of literacy level and previous exposure to terminology at prior appointments.

The patients were handed the questionnaire in the waiting room before their appointment. Many factors may have affected the quality and accuracy of answers, such as limited time before their appointment, anxiety and use of guessing or process of elimination on MCQs. A possible way to reduce limitations could be provided using a clinical kinematic assessment tool which the patient could complete at home. ${ }^{33}$ However, these come with their own limitations; for example, at home, the patient may seek help for answers from family, friends or other sources such as the internet, which they may not have done if monitored in the waiting room. Some patients may also struggle with the technical aspect of electronic forms and might not have the required support at home to access and complete the questionnaire.

Due to small sample size and anonymity of the questionnaire, the number of previous appointments, reason for attendance and socioeconomic factors (such as the Scottish Index of Multiple Deprivation and ethnicity) were not assessed. However, this paper highlights the importance of developing health literacy in dentistry and may provide a foundation for future research to expand on this topic.

Another area for future quality improvement projects or research could be to assess how the understanding of terminology differs in different specialities, such as in the restorative or paediatric departments. In addition, the questionnaire was only completed within one dental hospital; further questionnaires at multiple sites would lead to a larger and more diverse population sample, therefore providing a better overall insight, and this should be considered in future studies.

\section{Conclusion}

Patient understanding of terminology varies greatly and many factors influence this, including first language, education level, experience and demographics. It is crucial that clinicians take the time to establish each patient's health literacy level and adapt both their written and verbal communication as necessary to avoid concern, confusion and possible errors.
Acknowledgements

Ethics declaration: approval for this quality improvement project to improve communication in the Oral Medicine and Oral Surgery Department was granted by the Edinburgh Dental Institute audit and quality improvement team. The questionnaire was completely anonymised and stored on an NHS computer in the Edinburgh Dental Institute.

\section{Conflict of interest}

The authors declare no conflicts of interest.

\section{References}

1. Guadagnoli E, Ward P. Patient participation in decision-making. Soc Sci Med 1998; 47: 329-339.

2. Scambler S, Delgado M, Asimakopoulou K. Defining patient-centred care in dentistry? A systematic review of the dental literature. Br Dent J 2016; 221: 477-484

3. Fage-Butler A M, Nisbeth-Jensen M. Medical terminology in online patient-patient communication: evidence of high health literacy? Health Expect 2016; 19: 643-653.

4. Rayner H, Hickey M, Logan I, Mathers N, Rees $\mathrm{P}$ Shah R. Writing outpatient letters to patients. BMJ 2020; DOI: 10.1136/bmj.m24.

5. General Dental Council. Standards for the Dental Team. 2013. Available at https://www.gdc-uk.org/ docs/default-source/standards-for-the-dental-team/ standards-printer-friendly-black-white.pdf (accessed June 2020).

6. Andrus M R, Roth M T. Health literacy: a review. Pharmacotherapy 2002; 22: 282-302.

7. Henselmans I, Heijmans M, Rademakers J, Van Dulmen S. Participation of chronic patients in medical consultations: patients' perceived efficacy, barriers and interest in support. Health Expect 2015; 18: 2375-2388.

8. Academy of Medical Royal Colleges. Please, write to me. Writing outpatient letters to patients. Guidance. 2018. Available online at https://www.aomrc.org. uk/reports-guidance/please-write-to-me-writingoutpatient-clinic-letters-to-patients-guidance/ (accessed June 2020).

9. Hayes E, Dua R, Yeung E, Fan K. Patient understanding of commonly used oral medicine terminology. Br Dent $J$ 2018; 223: 842-845.

10. Blackman J, Sahebjalal M. Patient understanding of frequently used cardiology terminology. Br J Cardiol 2014; 1: 39.

11. Cosic F, Kimmel L, Edwards E. Patient Comprehension of Common Orthopedic Terminology. Health Lit Res Pract 2019; DOI: 10.3928/24748307-20190624-04.

12. O'Connell R L, Hartridge-Lambert S K, Din N, St John $E R$, Hitchins $C$, Johnson T. Patients' understanding of medical terminology used in the breast clinic. Breast 2013; 22: 836-838.

13. Peduzzi P, Concato J, Kemper E, Holford T R, Feinstein A R. A simulation study of the number of events per variable in logistic regression analysis. J Clin Epidemio 1996; 49: 1373-1379.

14. Cambridge Advanced Learner's Dictionary and Thesaurus. Jargon. Available at https://dictionary. cambridge.org/dictionary/english/jargon (accessed June 2020).

15. World Health Organisation. Health Promotion Glossary. 1998. Available at https://www.who.int/ healthpromotion/about/HPR\%20Glossary\%201998. pdf (accessed May 2020).

16. Stewart M A. Effective physician-patient communication and health outcomes: a review. Can Med Assoc J 1995; 152: 1423-1433.

17. Scottish Government. Scottish Survey of Adult Literacies 2009: Report of Findings. 2010. Available online at https://www.webarchive.org.uk/wayback/ archive/20170701074158/http://www.gov.scot/ Publications/2010/07/22091814/15 (accessed May 2020). 
18. Graham S, Brookey J. Do patients understand? Perm J 2008; 12: 67-69.

19. Parikh N S, Parker R M, Nurss J R, Baker D W, Williams $M V$. Shame and health literacy: the unspoken connection. Patient Educ Couns 1996; 27: 33-39.

20. Easton P, Entwistle V A, Williams B. How the stigma of low literacy can impair patient-professional spoken interactions and affect health: insights from a qualitative investigation. BMC Health Serv Res 2013; 13: 319

21. Cooke M W, Wilson S, Cox P. Public understanding of medical terminology: non-English speakers may not receive optimal care. Emerg Med J 2000; 17: 119-121.

22. Kessels R P. Patients' memory for medical information. J R Soc Med 2003; 96: 219-222.

23. Misra S, Daly B, Dunne S, Millar B, Packer M Asimakopoulou K. Dentist-patient communication: what do patients and dentists remember following a consultation? Implications for patient compliance. Patient Prefer Adher 2013: 7: 543-549.

24. Ley P. Communicating with patients: Improving communication, satisfaction and compliance. New York: Croom Helm, 1988

25. Lapakko D. Communication is $93 \%$ Nonverbal: An Urban Legend Proliferates. Commun Theatre Assoc Minn J 2007; 34: 7-19.

26. Mehrabian A, Ferris S R. Inference of attitudes from nonverbal communication in two channels. J Consult Psychol 1967; 31: 248-252.

27. Healthcare Improvement Scotland. Supporting personcentred care in COVID-19 situations. 2020. Available at https://ihub.scot/improvement-programmes/peopleled-care/person-centred-health-and-care/supportingperson-centred-care-in-covid-19-situations/ (accessed June 2020).

28. Marra A, Buonanno P, Vargas M. How COVID-19 pandemic changed our communication with families: losing nonverbal cues. Crit Care 2020; 24: 297.

29. O'Reilly M, Cahill M R, Perry I J. Writing to patients: a randomised controlled trial. Clin Med 2006; 6: 178-182.

30. NHS Digital service manual. Content style guide. 2019. Available at https://service-manual.nhs.uk/content (accessed May 2020).

31. Hong Y, Ehlers K, Gillis R, Patrick T, Zhang J. A usability study of patient-friendly terminology in an EMR system. Stud Health Technol Inform 2010; 160(Pt 1): $136-140$.

32. Ellis A, Fry R. Regional health inequalities in England. Reg Trends 2010; 42: 60-79.

33. Coats R O, Crossley K L, Conlin N et al. Cognitive and sensorimotor function in participants being treated for trigeminal neuralgia pain. J Headache Pain 2020 21: 91.

\section{Correction to: Research: Patient and clinician satisfaction with video consultations in dentistry - part one: patient satisfaction}

The original article can be found online at https://doi.org/10.1038/s41415-021-3007-y.

Author's correction note:

Research article Br Dent J 2021; https://doi.org/10.1038/s41415-021-3007-y

When this article was initially published the Acknowledgements were not included. The Acknowledgements section should have read:

The authors would like to thank: Julia Radecki, Renu George, Sara Stephens and Varlie Bacon for their assistance with this project.

The journal apologises for any inconvenience caused.

\section{Correction to: Research: Patient and clinician satisfaction with video consultations in dentistry - part two: clinician satisfaction}

The original article can be found online at https://doi.org/10.1038/s41415-021-3009-9.

Author's correction note:

Research article Br Dent J 2021; https://doi.org/10.1038/s41415-021-3009-9

When this article was initially published the Acknowledgements were not included. The Acknowledgements section should have read:

The authors would like to thank: Julia Radecki, Renu George, Sara Stephens and Varlie Bacon for their assistance with this project.

The journal apologises for any inconvenience caused. 